\title{
Cyclin D1 and P27KIP1: The Gatekeepers of Dysplasia
}

\author{
Mahmoud M. Bakr ${ }^{1}$, Simon Guan ${ }^{2}$, Norman Firth ${ }^{3}$, Robert M. Love ${ }^{1 *}$
}

'School of Dentistry and Oral Health, Griffith University, Australia

${ }^{2}$ School of Dentistry, University of Otago, New Zealand

${ }^{3}$ University of Queensland, Brisbane, Australia

\section{Article Info}

\section{Article Notes}

Received: May 08, 2018

Accepted: May 31, 2018

\section{*Correspondence:}

Prof. Robert M Love, Dean and Head of School, School of Dentistry and Oral Health, Griffith University, QLD 4222,

Australia;

Telephone: +61756780713

Email: r.love@griffith.edu.au

(C) 2018 Love RM. This article is distributed under the terms of the Creative Commons Attribution 4.0 International License.

\section{Keywords}

Cell cycle

Cyclin D1

Epithelial dysplasia

P27 K1P1

Cancer progression

\section{ABSTRACT}

There is increasing evidence suggesting that cyclins, cyclin-dependent kinases (CDKs), and cyclin-dependent kinase inhibitors (CDIs) either are themselves targets for genetic change in cancer or are disrupted secondarily by other oncogenic events. Cyclin D1 and $\mathrm{p} 27^{\mathrm{KIP} 1}$ are two important regulators at the G1/S checkpoint. Cyclin D1 is an oncogene of cell cycle regulation with positive effect. Normally, cyclin D1 at G1 is constant or at a very low level and its excessive expression may be associated with the disordered proliferation of cells leading to malignant change. On the other hand, $\mathrm{p} 27^{\mathrm{KIP} 1}$ is an antioncogene for cell cycle regulation, which functions as a negative regulator. Under the regulation of TGF- $\beta, p 27^{\mathrm{KIP} 1}$ inhibits the activity of oncogenes and controls the transition of the G1/S phase mainly by the interaction with CDK and CDK-Cyclin in order to inhibit cell proliferation and give cells opportunities to repair DNA. In addition, p27 $7^{\mathrm{KIP} 1}$ not only acts as CDK inhibitor, but also promotes cell differentiation and induces the apoptosis of cells. In this article we review studies that have explored the effects of cyclin D1 and P2 $7^{\mathrm{KIP} 1}$ on cancer progression and dysplasia with a specific focus on oral dysplasia and oral squamous cell carcinoma (OSCC). We also aim to shed some light on the different means of evaluating the interaction between Cyclin D1 and P2 $27^{\mathrm{KIP} 1}$ as well as the immunohistochemical reactions associated with different forms of cyclin D1.

\section{CELL CYCLE}

The cell cycle is the series of events that are required to create two daughter cells from a progenitor cell. The cell division cycle consists of four phases, i.e. G1, S, G2, and M. A cell that is not in the cell cycle is in a quiescent state named G0. G1 is the interval before DNA replication, $\mathrm{S}$ is the DNA replication phase, G2 is the interval after DNA replication, $\mathrm{M}$ is the mitotic phase. Progression through the cell cycle is governed by a family of cyclin-dependent kinases (CDKs), the activity of which is regulated by phosphorylation ${ }^{1}$, activated by binding of cyclins ${ }^{2}$, and inhibited by CDK inhibitors ${ }^{3}$.

The orderly progression through the different phases is assured by proteins that regulate critical checkpoints. Several checkpoints have been identified such as the late G1 phase restriction point, the G1/S phase transition, and the G2/M phase transition. These checkpoints normally verify that the preceding events have been completed before progressing to the next monitor completion of DNA replication and produce signals that interrupt the cell cycle in the event of an error or damage to the genome. Abnormal functioning of checkpoints, such as incapacity in detecting damaged DNA, may play a significant role in tumour progression by permitting cells to progress through the cell cycle with damaged or abnormal DNA ${ }^{4}$. 
The orderly progression of cells through the cell cycle is carefully orchestrated by CDK after they bind to a group of proteins called cyclins ${ }^{5}$. The cyclin/CDK complexes form heterodimers that phosphorylate many proteins involved in major cell cycle events ${ }^{6}$. The activity of cyclin/ CDK complexes is modulated by the phosphorylation of threonine residues on the $\mathrm{CDK}$, and by interactions with cyclin dependent kinase inhibitors (CDI). The proper and timely action of all these mechanisms is the basis of normal function, and consequently, dysregulation at many levels of the cell cycle has been implicated in tumourogenesis.

\section{CYCLINS AND CYCLIN D1}

Two major classes of cyclins are recognized according to the phase of action and to their pattern of degradation; these are the mitotic cyclins (Cyclin A, B) and the G1 cyclins (Cyclin A, D, E) ${ }^{7}$. Mitotic cyclins are essential for the control of the cell cycle at the G2/M transition (mitosis). They usually accumulate steadily during G2 and are abruptly destroyed as cell exit from mitosis. On the other hand, the G1 cyclins are essential for the control of the cell cycle at the G1/S transition. For example, cyclin A/CDK2 complex is active in $\mathrm{S}$ phase. Cyclin D/CDK4, cyclin D/CDK6, and cyclin $\mathrm{E} / \mathrm{CDK} 2$ regulate the transition from $\mathrm{G} 1$ to $\mathrm{S}$ phase $\mathrm{e}^{7,8}$.

There are three isoforms of cyclin D (Cyclin D1, D2, D3) in humans; cyclin D1 is perhaps the most studied D-type cyclin in human cancers ${ }^{9}$. In mice, cyclin D1 is located on chromosome 7. Human cyclin D1 is localized at chromosome $11 \mathrm{q} 13^{10}$. It is a nuclear protein that has been shown to be a key regulator of G1-S phase transition, and elevated levels of cyclin D1 induce apoptosis ${ }^{11}$. Cyclin D1 protein binds and activates CDK4 and CDK6, leading to phosphorylation of retinoblastoma protein (RB - a tumour suppressor protein) that results in release of transcriptional activator E2F, leading to transcription and activation of proteins associated with passage through the G1 check point and progression into the S phase ${ }^{9}$.

A review article states that cyclin D1 also acts as transcriptional modulator by regulating the activity of several transcription factors and histone deacetylase $\mathrm{e}^{12}$. This function is independent of the CDK4 activity. Cyclin D1 protein is unstable with a short half-life, about 24 minutes $^{13,14}$. It is degraded mainly by the $26 \mathrm{~S}$ proteasome in an ubiquitin-dependent pathway ${ }^{13}$. However, cyclin D1 is an important proto-oncogene. Overexpression of cyclin D1 leads to shortening of the G1 phase and to less dependency on exogenous mitogens, resulting in abnormal cell proliferation that in turn may favour the occurrence of additional genetic lesions ${ }^{15}$.

Cyclin D1 is reported as being overexpressed or amplified in a number of primary human cancers supporting its role as an oncogene. In many tumours, genetic alterations affecting the cyclin D1 gene frequently result in overexpression of cyclin D1 protein. Several studies have shown overexpression of cyclin D1 protein is associated with at least half of all invasive breast cancers $^{16-18}$. Many studies of mantle cell lymphoma have demonstrated increased activity in cyclin D19-21. It has been shown that overexpression of cyclin D1 by lymphocytes in the mantle zone impairs the capacity of these cells to exit the cell cycle and to differentiate into mature plasma cells ${ }^{22}$. Studies of oesophageal cancer also showed amplification and overexpression of cyclin D1 protein in $30 \%$ of the cases ${ }^{23,24}$. Amplification and increase expression of cyclin D1 protein have been observed in $10 \%$ of hepatocellular carcinoma ${ }^{25,26}$. Overexpression of cyclin D1 protein has also been associated with decreased survival and worse prognosis in different types of cancer including oesophageal squamous cell carcinoma, breast carcinoma and colonic adenocarcinoma ${ }^{27-29}$.

Furthermore, several studies have demonstrated that increased levels of cyclin D1 mRNA may be associated with decreased survival rate of patients with head and neck cancers $^{30-33}$. These observations suggest that cyclin D1 might play an important role in malignant transformation and disease progression. In addition to the above, over expression of cyclin D1 protein may be the consequence of gene rearrangement. Therefore, amplification of this gene often appears in malignant lesions ${ }^{34}$. Amplification of cyclin D1 gene has been demonstrated in $17 \%-55 \%$ of head and neck squamous cell carcinoma (HNSCC) in several studies $^{32,35,36}$. Over expression of cyclin D1 protein has also been shown in 21\%-64\% of HNSCC and associated with a poor prognosis, more frequent recurrence, and shorter time to recurrence ${ }^{30,37-40}$. Moreover, overexpression has been shown to be associated with lymph node metastasis ${ }^{38}$. Little is known of the frequency and the timing of this change in oral epithelial dysplasia ${ }^{41}$. Overexpression of cyclin D1 in oral epithelial dysplasia has been reported in some studies ${ }^{41-43}$. There are some studies on cyclin D1 degradation in vitro by a number of therapeutic agents. They showed that induction of cyclin D1 degradation might offer a useful avenue for therapeutic intervention ${ }^{4-49}$. A summary of studies (1990-2018) on expression of cyclin D1 and CDKs in normal tissues, oral epithelial dysplasia and squamous cell carcinoma as well as other different types of cancer is shown in Table 1.

\section{CYCLIN DEPENDENT KINASES and P2 $7^{\mathrm{KIP} 1}$}

Cyclin-dependent kinases (CDKs) are a family of protein kinases that are involved in regulating the cell cycle. Their activity is controlled by a complex network of regulatory subunits and phosphorylation events. In the cell, these regulatory mechanisms generate an interlinked series of CDK oscillators that trigger the events of cell division. Therefore, they may be considered as the engines that drive the events of the cell cycle ${ }^{50}$. There are at least nine 
different cyclin-dependent kinases in eukaryotic cells, four of which, CDK1, 2, 3, and 4, have been shown to play an important role in the regulation of the eukaryotic cell division cycle and have also been suggested in the control of gene transcription and other processes ${ }^{50}$.

Cyclin-dependent kinase inhibitors (CDIs) are proteins that inhibit cyclin-dependent kinase. Cell cycle progression is negatively controlled by CDIs. They are involved in the cell cycle arrest at the G1 phase. Two families of CDIs negatively regulate $\mathrm{CDK}$ activities and mediate cell cycle arrest following growth inhibitory stimuli ${ }^{51}$. The INhibitors of CDK4, also known as the INK4 family members involve $\mathrm{p} 15^{\mathrm{INK} 4 \mathrm{~B}}, \mathrm{p} 16^{\mathrm{INK} 4 \mathrm{~A}}, \mathrm{p} 18^{\mathrm{INK} 4 \mathrm{C}}$, and $\mathrm{p} 19^{\mathrm{INK} 4 \mathrm{D}}$. They specifically inhibit cyclin D1 associated kinase ${ }^{52,53}$. Members of the kinase inhibitor protein (KIP) family involve P21 $1^{\mathrm{CIP} 1}$ or CDK-interacting protein 1 , also known asP21 $1^{\text {WAF1 }}, \mathrm{P} 21^{\text {SDI } 1}$ or senescence DNA synthesis inhibitor $1, \mathrm{P} 27^{\mathrm{KIP} 1}$ or kinase inhibitor protein 1 , and $\mathrm{P} 57^{\mathrm{KIP} 2}$ or kinase inhibitor protein 2. It has been suggested that they bind and inhibit cyclin D/ CDK4, cyclin E/CDK2 and cyclin A/CDK2 complexes ${ }^{51}$. P21 is a potent CDI. It is a major element in cell cycle control and it is mainly regulated at the transcriptional level. It binds to and inhibits the activity of cyclin E/CDK2, cyclin $\mathrm{B} / \mathrm{CDK} 1$ and cyclin D/CDK4/6 complexes and thus acts as a regulator of cell cycle progression at G1/S phase ${ }^{54}$.

P27 $7^{\mathrm{KIP} 1}$ is a cyclin-dependent kinase inhibitor and a tumour suppressor that regulates G0 to $S$ phase transition $s^{55}$. It has been identified as an inhibitor in cells arrested by transforming growth factor $-\beta$ (TGF- $\beta$ ) and is regulated by growth inhibitory cytokines and by contact inhibition $^{56-58}$. P2 $27^{\mathrm{KIP} 1}$ protein is strongly expressed in non-proliferating cells and plays an important role in the regulation of both quiescence and G1 progression ${ }^{59}$.

$\mathrm{P} 27^{\mathrm{KIP} 1}$ 's inhibitory activity is mainly controlled by its concentration, subcellular localization and phosphorylation status ${ }^{55}$. The levels and activity of $\mathrm{p} 27^{\mathrm{KIP} 1}$ protein increase in response to a number of factors, including cell density, differentiation signals, following loss of adhesion to the extracellular matrix, and in response to growth inhibitory signaling by TGF- $\beta$ or the drug lovastatin ${ }^{60-63}$. P2 $27^{\mathrm{KIP} 1}$ is not a classic tumour suppressor like P53, but loss of p27 KIP1 protein could result in resistance to growth inhibitory factors, deregulation of cell proliferation, and oncogenic change $e^{64}$.

In quiescent normal epithelia of breast, prostate, ovary, lung and other sites, $\mathrm{p} 27^{\mathrm{KIP} 1}$ protein is expressed at high level, and loss or reduction of the level of this protein may be seen in carcinomas ${ }^{59}$. When both a non-invasive and invasive component coexist in the tumour, loss of p2 $7^{\mathrm{KIP} 1}$ protein is detected in both carcinoma in situ and invasive tumour, suggesting that events leading to deregulation of $\mathrm{p} 27^{\mathrm{KIP} 1}$ protein may precede invasion ${ }^{59}$. For example, $\mathrm{p} 27^{\mathrm{KIP} 1}$ protein is reduced in benign prostatic hypertrophy, a hyperplastic premalignant prostatic neoplasm ${ }^{65-67}$. Study of prostate carcinoma also showed variable degrees of reduction in $\mathrm{p} 27^{\mathrm{KIP} 1}$ staining was frequently observed in the prostatic intraepithelial neoplasia adjacent to invasive carcinoma ${ }^{66}$. Studies of breast cancer also have similar results; $27^{\mathrm{KIP} 1}$ protein is reduced in premalignant and non-invasive malignant lesions, including ductal carcinoma in situ of the breast ${ }^{68-71}$. In addition, a comparison of $\mathrm{p} 27^{\mathrm{KIP} 1}$ protein levels in primary colon carcinoma and metastatic tumour demonstrated a reduction of $\mathrm{p} 27^{\mathrm{KIP} 1}$ staining in the metastatic tumour ${ }^{72,73}$. Therefore, reduction in p2 $7^{\mathrm{KIP} 1}$ protein level may contribute to cancer progression in the transitions from carcinoma in situ to invasive tumour, and from localized primary tumour to metastatic tumour ${ }^{74}$.

Moreover, decreased levels of p27 ${ }^{\mathrm{KI} 1}$ protein may be related to high tumour grade and stage in human colorectal $^{64}$, gastric ${ }^{75}$, breast $^{76}$, prostate ${ }^{66,77}$ and other cancers. Reduction of $\mathrm{p} 27^{\mathrm{KIP} 1}$ protein in tumours correlates significantly with decreased survival in colorectal $^{78}$, gastric $^{79,80}$, breast ${ }^{76,81,82}$ and esophageal ${ }^{83}$ squamous cell carcinoma patients, among others. These studies have suggested that $\mathrm{p} 27^{\mathrm{KIP} 1}$ plays an important role in tumour suppression. In fact, identification of the $\mathrm{p} 27^{\mathrm{KIP} 1}$ proteolysis pathways has opened new avenues for therapeutic intervention in cancer ${ }^{84}$. A summary of studies (1990-2018) on expression of cyclin D1 and CDKs in normal tissues, oral epithelial dysplasia and squamous cell carcinoma as well as other different types of cancer is shown in Table 1.

\section{IMMUNOHISTOCHEMICAL EVALUATION OF THE INTERACTION BETWEEN CYCLIN D1 AND P27 ${ }^{\mathrm{KIP} 1}$}

In most studies, the immunohistochemical reactivity for cyclin D1 and p27 $7^{\mathrm{KIP} 1}$ was evaluated on the basis of presence or absence of nuclear and/or cytoplasmic staining ${ }^{90,104,107-109}$. However, Gillett et al. excluded cyclin D1 cytoplasmic staining alone in their cases and considered it as negative ${ }^{110}$. It has been shown that cyclin D1 plays an important role in cell proliferation and differentiation and can be transported between nuclear and cytoplasmic compartments via nuclear pores during different phases of the cell cycle ${ }^{111}$.

Most of the previous studies suggest cyclin D1 is a nuclear protein ${ }^{112-114}$. However, results from Guan et al., $2018^{104}$ (strong nuclear and cytoplasmic staining) imply that for proteins such as cyclin D1, it is more accurate to apply to nuclear/cytoplasmic distribution ratio than a single cellular localization. Moreover, the capacity of the cell to direct nuclear import and export during cell cycle suggested that movement of cyclin D1 must be considered bi-directional ${ }^{115}$. The redistribution of cyclin D1 protein correlates with its phosphorylation on Thr-286 by 
Table 1: Illustrates a summary of studies (1990-2018) that investigated the expression of cyclin D1 and CDKs in normal tissues, oral epithelial dysplasia and squamous cell carcinoma as well as other different types of cancer.

\begin{tabular}{|c|c|c|c|c|c|}
\hline $\begin{array}{l}\text { Bibliographic } \\
\text { citation }\end{array}$ & Study type & Number of patients & Case/setting & $\begin{array}{l}\text { Marker } \\
\text { protein }\end{array}$ & Effect size \\
\hline $\begin{array}{c}\text { (Michalides et al., } \\
1995)^{30}\end{array}$ & $\begin{array}{l}\text { Retrospective case- } \\
\text { control pathology } \\
\text { slides }\end{array}$ & $\begin{array}{l}47 \text { Head and Neck } \\
\text { Squamous Cell } \\
\text { Carcinomas }\end{array}$ & $\begin{array}{l}\text { Random selection of } \\
\text { cases. Single centre } \\
\text { study. Netherlands }\end{array}$ & Cyclin D1 & $\begin{array}{c}\text { Overexpression of cyclin D1 } \\
\text { associated with poor prognosis } \\
\text { and a shortened overall survival of } \\
\text { these patients ( } \mathrm{P}=0.0095) \text {. }\end{array}$ \\
\hline $\begin{array}{c}\text { (Akervall et al., } \\
\text { 1997) }\end{array}$ & $\begin{array}{l}\text { Retrospective case } \\
\text { control pathology } \\
\text { slides }\end{array}$ & $\begin{array}{l}75 \text { Head and Neck } \\
\text { Squamous Cell } \\
\text { Carcinomas }\end{array}$ & $\begin{array}{l}\text { Random selection. } \\
\text { Matched for site. Dual } \\
\text { centre. Sweden and } \\
\text { Netherlands }\end{array}$ & Cyclin D1 & $\begin{array}{l}\text { Patients with tumors strongly } \\
\text { positive for Cyclin D1 had poorer } \\
\text { survival }(P=0.047) \text {. }\end{array}$ \\
\hline (Xu et al., 1998) & $\begin{array}{l}\text { Retrospective case } \\
\text { control pathology } \\
\text { slides }\end{array}$ & $\begin{array}{l}34 \text { Oral Squamous } \\
\text { Cell Carcinomas } \\
2 \text { Oral Epithelial } \\
\text { Dysplasia }\end{array}$ & $\begin{array}{c}\text { Random selection. } \\
\text { Matched for site and } \\
\text { Grade. Dual centre. Texas } \\
\text { and Argentina }\end{array}$ & $\begin{array}{l}\text { P53, Cyclin } \\
\text { D1, Rb and } \\
\text { H-ras }\end{array}$ & $\begin{array}{l}\text { High frequency (41\%) of Cyclin D1 } \\
\text { Overexpression }\end{array}$ \\
\hline $\begin{array}{l}\text { (Castle et al., } \\
\text { 1999) }^{42}\end{array}$ & $\begin{array}{l}\text { Retrospective case } \\
\text { control pathology } \\
\text { slides }\end{array}$ & $\begin{array}{l}25 \text { Oral Dysplasia } \\
47 \text { Oral Squamous } \\
\text { Cell Carcinomas }\end{array}$ & $\begin{array}{l}\text { Random selection } \\
\text { Matched for site, grade, } \\
\text { age, gender. Single } \\
\text { centre. Florida. }\end{array}$ & $\begin{array}{l}\text { P53 and } \\
\text { Cyclin D1 }\end{array}$ & $\begin{array}{l}\text { Overexpression of Cyclin D1 was } \\
\text { not significant different between } \\
2 \text { age groups studies either for } \\
\text { dysplasia or carcinomas. }\end{array}$ \\
\hline $\begin{array}{c}\text { (Bova et al., } \\
1999)^{38}\end{array}$ & $\begin{array}{l}\text { Retrospective case } \\
\text { control pathology } \\
\text { slides }\end{array}$ & $\begin{array}{l}147 \text { Carcinoma of } \\
\text { the Anterior Tongue }\end{array}$ & $\begin{array}{l}\text { Random selection } \\
\text { Matched for site, grade, } \\
\text { age, gender. Single } \\
\text { centre. Australia. }\end{array}$ & $\begin{array}{c}\text { Cyclin D1 } \\
\text { and P16 } 16^{\text {INK4A }}\end{array}$ & $\begin{array}{l}\text { Overexpression of Cyclin D1 } \\
\text { occurred in } 68 \% \text { of tumors and was } \\
\text { associated with increased lymph } \\
\text { node stage }(P=0.014) \text {, increased } \\
\text { tumor grade }(P=0.003) \text {, and } \\
\text { reduced disease-free }(P=0.006) \text { and } \\
\text { overall }(P=0.01) .\end{array}$ \\
\hline $\begin{array}{l}\text { (Lam et al., } \\
2000)^{86}\end{array}$ & $\begin{array}{l}\text { Retrospective case } \\
\text { control pathology } \\
\text { slides }\end{array}$ & $\begin{array}{l}56 \text { Oral Squamous } \\
\text { Cell Carcinomas }\end{array}$ & $\begin{array}{l}\text { Random selection } \\
\text { Matched for site, grade, } \\
\text { age, gender. Single } \\
\text { centre. Hong Kong. }\end{array}$ & $\begin{array}{l}\text { P53 and } \\
\text { Cyclin D1 }\end{array}$ & $\begin{array}{l}\text { Cyclin D1 expression was found } \\
\text { in } 63 \% \text { of Oral Squamous Cell } \\
\text { Carcinomas and was more } \\
\text { frequently positive in high-grade } \\
\text { lesions ( } P=0.019)\end{array}$ \\
\hline $\begin{array}{c}\text { (Nakahara et al., } \\
2000)^{87}\end{array}$ & $\begin{array}{l}\text { Retrospective case } \\
\text { control pathology } \\
\text { slides }\end{array}$ & $\begin{array}{l}78 \text { Oral Squamous } \\
\text { Cell Carcinomas } \\
46 \text { Leukoplakia } \\
20 \text { Normal mucosa }\end{array}$ & $\begin{array}{l}\text { Random selection } \\
\text { Matched for site, grade, } \\
\text { age, gender. Single } \\
\text { centre. Japan. }\end{array}$ & $\begin{array}{c}\text { Cyclin D1 } \\
\text { and P16 } 16^{\text {INK4A }}\end{array}$ & $\begin{array}{l}\text { The Overexpression of Cyclin } \\
\text { D1 was not Observed in normal } \\
\text { mucosa and was observed in } 35.9 \% \\
\text { of Squamous Cell Carcinomas }\end{array}$ \\
\hline $\begin{array}{l}\text { (Mineta et al., } \\
\text { 2000) }^{39}\end{array}$ & $\begin{array}{l}\text { Retrospective case } \\
\text { control pathology } \\
\text { slides }\end{array}$ & $\begin{array}{l}94 \text { Tongue } \\
\text { Squamous Cell } \\
\text { Carcinomas }\end{array}$ & \begin{tabular}{|} 
Random selection \\
Matched for Age, \\
Gender, Smoking Alcohol \\
and grade. Dual centre. \\
Japan and Sweden.
\end{tabular} & Cyclin D1 & $\begin{array}{l}\text { 19\% of patients showed Cyclin D1 } \\
\text { overexpression. The 5- year survival } \\
\text { rate of high Cyclin expressors was } \\
39 \%(P=0.04) .\end{array}$ \\
\hline $\begin{array}{c}\text { (Rousseau et al., } \\
2001)^{41}\end{array}$ & $\begin{array}{l}\text { Retrospective case } \\
\text { control pathology } \\
\text { slides }\end{array}$ & $\begin{array}{l}20 \text { Normal Mucosa, } \\
22 \text { Mild Epithelial } \\
\text { Dysplasia, } 20 \\
\text { Moderate Epithelial } \\
\text { Dysplasia, } 17 \\
\text { Severe Epithelial } \\
\text { Dysplasia and } 25 \\
\text { Oral Squamous Cell } \\
\text { Carcinoma }\end{array}$ & $\begin{array}{l}\text { Random selection } \\
\text { Matched for grade. Dual } \\
\text { centre. Canada and USA. }\end{array}$ & Cyclin D1 & $\begin{array}{l}\text { Overexpression of Cyclin D1 was } \\
\text { identified in } 29 \% \text { of mild, } 47 \% \\
\text { moderate, } 29 \% \text { of severe Oral } \\
\text { epithelial dysplasia. There were } \\
\text { statistically significant correlations } \\
\text { identified between gene and } \\
\text { protein levels in all categories of } \\
\text { disease. }\end{array}$ \\
\hline $\begin{array}{l}\text { (Ronaldo et al., } \\
\text { 2001) }\end{array}$ & $\begin{array}{l}\text { Retrospective case } \\
\text { control pathology } \\
\text { slides }\end{array}$ & $\begin{array}{l}112 \text { Carcinoma of } \\
\text { the Anterior Tongue }\end{array}$ & $\begin{array}{l}\text { Random selection. } \\
\text { Matched for site, grade, } \\
\text { age, gender. Single } \\
\text { centre. Australia. }\end{array}$ & Cyclin D1 & $\begin{array}{l}\text { Overexpression of cyclin D1 ( } 65 \% \\
\text { of the cases) associated with poor } \\
\text { prognosis. }\end{array}$ \\
\hline $\begin{array}{c}\text { (Sathyan et al., } \\
\text { 2006) }\end{array}$ & $\begin{array}{l}\text { Retrospective case } \\
\text { control pathology } \\
\text { slides }\end{array}$ & $\begin{array}{l}147 \text { Buccal } \\
\text { Squamous Cell } \\
\text { Carcinoma and } 94 \\
\text { Tongue Squamous } \\
\text { Cell Carcinoma }\end{array}$ & $\begin{array}{l}\text { Random selection. } \\
\text { Matched for site, grade, } \\
\text { age, gender. Single } \\
\text { centre. India }\end{array}$ & $\begin{array}{l}\text { P53, Rb, P16, } \\
\text { Cyclin D1, } \\
\text { CDK4 and } \\
\text { PCNA }\end{array}$ & $\begin{array}{l}\text { Among the biological markers, } \\
\text { the Overexpression of CyclinD } 1 \\
(P=0.007) \text { showed significant } \\
\text { association with shorter disease- } \\
\text { free survival in these cases. }\end{array}$ \\
\hline
\end{tabular}




\begin{tabular}{|c|c|c|c|c|c|}
\hline $\begin{array}{c}\text { (Kövesi \& Szende, } \\
2006)^{90}\end{array}$ & $\begin{array}{l}\text { Retrospective case } \\
\text { control pathology } \\
\text { slides }\end{array}$ & 18 Oral Leukoplakia & $\begin{array}{l}\text { Random selection. } \\
\text { Matched for smoking, } \\
\text { alcohol, grade, age and } \\
\text { gender. Single centre. } \\
\text { Hungary }\end{array}$ & $\begin{array}{l}\text { Cyclin D1, } \\
\text { P27 } 7^{\mathrm{KIP} 1} \text { and } \\
\text { P63 }\end{array}$ & $\begin{array}{l}\text { The severity of dysplasia showed } \\
\text { positive correlation with the } \\
\text { severity of Leukoplasia }\end{array}$ \\
\hline $\begin{array}{l}\text { (Matsushima et } \\
\text { al., 2006) }\end{array}$ & $\begin{array}{l}\text { Retrospective case } \\
\text { control pathology } \\
\text { slides }\end{array}$ & $\begin{array}{l}84 \text { Nasopahrungeal } \\
\text { Carcinoma } \\
28 \text { Mild Epithelial } \\
\text { Dysplasia, } 28 \text { Mild } \\
\text { Moderate Epithelial } \\
\text { Dysplasia, and } 28 \\
\text { Moderate Epithelial } \\
\text { Dysplasia }\end{array}$ & $\begin{array}{l}\text { Random selection. } \\
\text { Matched for grade. } \\
\text { Single centre. Japan. }\end{array}$ & $\begin{array}{c}\text { Cyclin D1 and } \\
\text { Cyclin B1 }\end{array}$ & $\begin{array}{l}\text { The expression of Cyclin D1 } \\
\text { correlates with the grade of } \\
\text { Epithelial Dysplasia. }\end{array}$ \\
\hline $\begin{array}{l}\text { (Angadi \& } \\
\text { Krishnapillai, } \\
2007)^{92}\end{array}$ & $\begin{array}{l}\text { Retrospective case } \\
\text { control pathology } \\
\text { slides }\end{array}$ & $\begin{array}{l}71 \text { cases of } \\
\text { Oral Squamous } \\
\text { Cell Carcinoma } \\
\text { and Verrucous } \\
\text { Carcinoma }\end{array}$ & $\begin{array}{l}\text { Random selection. } \\
\text { Matched for grade. } \\
\text { Single centre. India. }\end{array}$ & Cyclin D1 & $\begin{array}{c}\text { Overexpression of Cyclin D1 } \\
\text { significantly correlated with lack of } \\
\text { differentiation in these malignant } \\
\text { epithelial neoplasms. }\end{array}$ \\
\hline $\begin{array}{l}\text { (Huang et al., } \\
\text { 2012) }\end{array}$ & $\begin{array}{l}\text { Retrospective case } \\
\text { control pathology } \\
\text { slides }\end{array}$ & $\begin{array}{l}264 \text { Oral Squamous } \\
\text { Cell Carcinoma }\end{array}$ & $\begin{array}{c}\text { Random selection. Single } \\
\text { Centre, Taiwan }\end{array}$ & Cyclin D1 & $\begin{array}{l}\text { Overexpression of cyclin D1 was } \\
\text { found in ( } 36.7 \%) \text { OSCCs and was } \\
\text { associated with poor clinical } \\
\text { outcomes. }\end{array}$ \\
\hline $\begin{array}{l}\text { (Uma et al., } \\
2012)^{93}\end{array}$ & $\begin{array}{l}\text { Retrospective case } \\
\text { control pathology } \\
\text { slides }\end{array}$ & $\begin{array}{l}20 \text { Oral Squamous } \\
\text { Cell Carcinoma and } \\
10 \text { Normal Mucosa }\end{array}$ & $\begin{array}{c}\text { Random selection. Single } \\
\text { Centre, India }\end{array}$ & $\begin{array}{l}\text { P53 and } \\
\text { Cyclin D1 }\end{array}$ & $\begin{array}{l}\text { Increased Cyclin D1 expression } \\
\text { was seen in Oral Squamous Cell } \\
\text { Carcinoma when compared to } \\
\text { the normal mucosa and a positive } \\
\text { correlation was seen in increased } \\
\text { Cyclin D1 in OSCC. }\end{array}$ \\
\hline (Liu et al., 2013) $^{94}$ & $\begin{array}{l}\text { Retrospective case } \\
\text { control pathology } \\
\text { slides }\end{array}$ & $\begin{array}{l}130 \text { Primary } \\
\text { Nasopharyngeal } \\
\text { Carcinoma }\end{array}$ & $\begin{array}{c}\text { Random selection. Single } \\
\text { Centre, China }\end{array}$ & $\mathrm{P} 27^{\mathrm{KIP} 1}$ & $\begin{array}{l}\text { Nuclear expression of p } 27^{\mathrm{KIP} 1} \\
\text { was inversely correlated with T } \\
\text { classification and clinical stage. } \\
\text { Patients with nuclear p } 27^{\mathrm{KIP} 1} \\
\text { expression had better overall } \\
\text { survival rates. }\end{array}$ \\
\hline (Li et al., 2014) ${ }^{95}$ & $\begin{array}{c}\text { Meta-analysis of } \\
\text { observational studies }\end{array}$ & $\begin{array}{l}4150 \text { cases of } \\
\text { colorectal cancer } \\
\text { from } 22 \text { studies }\end{array}$ & $\begin{array}{l}\text { Comprehensive literature } \\
\text { search for relevant } \\
\text { studies published } \\
\text { was performed using } \\
\text { PubMed, EMBASE, and } \\
\text { ISI Web of Science. }\end{array}$ & Cyclin D1 & $\begin{array}{l}\text { Cyclin D1 overexpression is an } \\
\text { unfavorable prognostic factor for } \\
\text { CRC. Cyclin D1 overexpression } \\
\text { might be associated with poor } \\
\text { clinical outcome and some } \\
\text { clinicopathological factors such as } \\
\text { age, T category, N category and } \\
\text { distant metastasis in CRC patients. }\end{array}$ \\
\hline $\begin{array}{l}\text { (Pereira et al., } \\
\text { 2014) }\end{array}$ & $\begin{array}{l}\text { Retrospective case } \\
\text { control pathology } \\
\text { slides }\end{array}$ & $\begin{array}{c}85 \text { Prostate } \\
\text { Carcinoma } 10 \\
\text { Normal prostate } \\
\text { tissue }\end{array}$ & $\begin{array}{c}\text { Random selection. Single } \\
\text { Centre, Brazil. }\end{array}$ & Cyclin D1 & $\begin{array}{l}\text { High cyclin D1 expression could } \\
\text { be a potential marker for tumor } \\
\text { aggressiveness. }\end{array}$ \\
\hline $\begin{array}{c}\text { (Ravikumar and } \\
\text { Ananthamurthy, } \\
2014)^{97}\end{array}$ & $\begin{array}{l}\text { Retrospective case } \\
\text { control pathology } \\
\text { slides }\end{array}$ & $\begin{array}{l}35 \text { Ducal Carcinoma } \\
\text { of the breast }\end{array}$ & $\begin{array}{c}\text { Random selection. Single } \\
\text { Centre, India. }\end{array}$ & Cyclin D1 & $\begin{array}{l}\text { Cyclin D1 expression was seen in } \\
67.5 \% \text { of ductal carcinoma }\end{array}$ \\
\hline $\begin{array}{l}\text { (Moreno-Galido } \\
\text { et al., 2014) }\end{array}$ & $\begin{array}{l}\text { Retrospective case } \\
\text { control pathology } \\
\text { slides }\end{array}$ & $\begin{array}{l}41 \text { Primary } \\
\text { Laryngeal / } \\
\text { Hypopharyngeal } \\
\text { Squamous Cell } \\
\text { Carcinima }\end{array}$ & $\begin{array}{l}\text { Random selection. } \\
\text { Matched for induction } \\
\text { chemotherapy } \\
\text { treatment. Single Centre, } \\
\text { Spain. }\end{array}$ & $\begin{array}{l}\text { EGFR, p53, } \\
\text { Cyclin D1, } \\
\text { p16, p21, } \\
\text { p27 }{ }^{\mathrm{KIP} 1} \\
\text { p-AKT, HIF- } \\
1 \alpha, \text { Caspase } \\
3 \text { and BCL2 }\end{array}$ & $\begin{array}{l}\text { Positive expression of } \mathrm{p} 27^{\mathrm{KIP} 1} \text { and } \\
\mathrm{BCL} 2 \text { had a significant predictive } \\
\text { value for chemotherapy response } \\
\text { in univariate analysis. } \mathrm{P} 27^{\mathrm{KIP} 1} \\
\text { expression was the only significant } \\
\text { predictor of chemotherapy } \\
\text { response in multivariate analysis } \\
(\mathrm{P}=0.015) \text {. }\end{array}$ \\
\hline
\end{tabular}




\begin{tabular}{|c|c|c|c|c|c|}
\hline $\begin{array}{l}\text { (Malgaonkar et } \\
\text { al., 2016) }\end{array}$ & $\begin{array}{l}\text { Retrospective case } \\
\text { control pathology } \\
\text { slides }\end{array}$ & $\begin{array}{l}19 \text { Pleomorphic } \\
\text { Adenoma, } 8 \\
\text { Mucoepidermoid } \\
\text { Carcinoma and } \\
7 \text { Adenoid Cystic } \\
\text { Carcinoma }\end{array}$ & $\begin{array}{l}\text { Random selection. Single } \\
\text { Centre, Saudi Arabia. }\end{array}$ & $\mathrm{P} 27^{\mathrm{KIP} 1}$ & $\begin{array}{l}52.6 \% \text { of pleomorphic adenoma } \\
\text { cases, } 25 \% \text { of mucoepidermoid } \\
\text { carcinoma cases and only } 14.2 \% \\
\text { of adenoid cystic carcinoma } \\
\text { cases showed strong expression } \\
\text { suggesting variable p } 27^{\mathrm{KIP} 1} \\
\text { expression in both malignant } \\
\text { neoplasms. }\end{array}$ \\
\hline $\begin{array}{c}\text { (Khabaz et al., } \\
\text { 2016) }\end{array}$ & $\begin{array}{l}\text { Retrospective case } \\
\text { control pathology } \\
\text { slides }\end{array}$ & $\begin{array}{l}124 \text { Bladder Cancer } \\
\text { and } 24 \text { Normal } \\
\text { Bladder Tissue }\end{array}$ & $\begin{array}{c}\text { Random selection. Single } \\
\text { Centre, Saudi Arabia. }\end{array}$ & Cyclin D1 & $\begin{array}{c}\text { Strong cyclin D1 } \\
\text { immunohistochemical staining } \\
\text { has been significantly linked with } \\
\text { low grades }(P=0.001) \text {, low stages } \\
\text { ( } P=0.005) \text {. Cyclin } D 1 \text { maybe a } \\
\text { valuable tissue biomarker for } \\
\text { presaging grade, stage, and poor } \\
\text { prognosis in bladder cancer. }\end{array}$ \\
\hline $\begin{array}{l}\text { (Dhingra et al., } \\
\text { 2017) }^{101}\end{array}$ & $\begin{array}{l}\text { Retrospective case } \\
\text { control pathology } \\
\text { slides }\end{array}$ & $\begin{array}{l}48 \text { Head and Neck } \\
\text { Squamous Cell } \\
\text { Carcinoma (HNSCC) }\end{array}$ & $\begin{array}{c}\text { Random selection. Single } \\
\text { Centre, India. }\end{array}$ & Cyclin D1 & $\begin{array}{l}\text { A significant association was seen } \\
\text { between Cyclin D1 expression with } \\
\text { tumour stage and with lymph node } \\
\text { metastasis but not with grade. }\end{array}$ \\
\hline $\begin{array}{l}\text { (Barić et al., } \\
\text { 2017) }\end{array}$ & $\begin{array}{l}\text { Retrospective case } \\
\text { control pathology } \\
\text { slides }\end{array}$ & $\begin{array}{c}70 \text { Papillary Thyroid } \\
\text { Microcarcinoma }\end{array}$ & $\begin{array}{c}\text { Random selection. Single } \\
\text { Centre, Croatia. }\end{array}$ & $\begin{array}{l}\text { Cyclin D1, } \\
\text { P27 }{ }^{\mathrm{KIP} 1} \text { and } \\
\text { RET }\end{array}$ & $\begin{array}{l}\text { Cyclin D1 and RET expression is } \\
\text { not crucial for the development } \\
\text { of metastases in lymph nodes. } \\
\text { In contrast, p27 } 27^{\mathrm{KP} 1} \text { expression is } \\
\text { significantly associated with lymph } \\
\text { node metastasis. }\end{array}$ \\
\hline $\begin{array}{l}\text { (Patel et al., } \\
\text { 2017) }\end{array}$ & $\begin{array}{l}\text { Retrospective case } \\
\text { control pathology } \\
\text { slides }\end{array}$ & $\begin{array}{l}30 \text { Oral Squamous } \\
\text { Cell Carcinoma and } \\
30 \text { Leukoplakia }\end{array}$ & $\begin{array}{c}\text { Random selection. Single } \\
\text { Centre, India. }\end{array}$ & $\begin{array}{l}\text { Cyclin D1 } \\
\text { and P63 }\end{array}$ & $\begin{array}{l}\text { The overall expression of cyclin } \\
\text { D1 and p63 correlated with tumor } \\
\text { differentiation, poor histological } \\
\text { grades, from well-differentiated to } \\
\text { poorly-differentiated SCC and the } \\
\text { severity of leukoplakia. }\end{array}$ \\
\hline $\begin{array}{l}\text { (Guan et al., } \\
2018)^{104}\end{array}$ & $\begin{array}{l}\text { Retrospective case } \\
\text { control pathology } \\
\text { slides }\end{array}$ & $\begin{array}{c}10 \text { Normal } \\
\text { Mucosa, } 12 \text { Mild to } \\
\text { Moderate Epithelial } \\
\text { Dysplasia, and } 11 \\
\text { Oral Squamous Cell } \\
\text { Carcinoma }\end{array}$ & $\begin{array}{c}\text { Random selection. Single } \\
\text { Centre, Dunedin, New } \\
\text { Zealand }\end{array}$ & $\begin{array}{l}\text { Cyclin D1 } \\
\text { and P27 }\end{array}$ & $\begin{array}{l}\text { A significant increase in expression } \\
\text { of cyclin D1 and a decrease in } \\
\text { expression of } \mathrm{p} 27^{\mathrm{KIP} 1} \text { proteins were } \\
\text { observed in oral epithelial dysplasia } \\
\text { and less differentiated OSCC. The } \\
\text { characteristic expression of both } \\
\text { cyclin D1 and p } 27^{\mathrm{KIP} 1} \text { correlate } \\
\text { with the grade of oral epithelial } \\
\text { dysplasia and degree of OSCC } \\
\text { differentiation. }\end{array}$ \\
\hline $\begin{array}{c}\text { (Ramos-Garcia et } \\
\text { al., 2018) }\end{array}$ & $\begin{array}{l}\text { Retrospective case } \\
\text { control pathology } \\
\text { slides }\end{array}$ & $\begin{array}{l}69 \text { Oral Squamous } \\
\text { Cell Carcinoma }\end{array}$ & $\begin{array}{c}\text { Random selection. Single } \\
\text { Centre, Spain. }\end{array}$ & $\begin{array}{l}\text { Cyclin D1 } \\
\text { and Ki-67 }\end{array}$ & $\begin{array}{l}\text { Cytoplasmic cyclin D1 expression } \\
\text { was associated with advanced } \\
\text { tumor stage, poor differentiation, } \\
\text { elevated Ki-67 expression, and } \\
\text { the presence of invasive cell } \\
\text { morphology, indicators of a poor } \\
\text { prognosis. }\end{array}$ \\
\hline $\begin{array}{l}\text { (Filipits et al., } \\
2018)^{106}\end{array}$ & $\begin{array}{l}\text { Retrospective case } \\
\text { control pathology } \\
\text { slides }\end{array}$ & $\begin{array}{l}862 \text { Early Breast } \\
\text { Cancer }\end{array}$ & $\begin{array}{l}\text { Random selection. } \\
\text { Matched for } \\
\text { Trastuzumab treatment. } \\
\text { Multiple Centres. } \\
\text { Austria, Canada, } \\
\text { Germany, Australia and } \\
\text { Greece. }\end{array}$ & $\begin{array}{l}\text { TOP2A, Ki67, } \\
\text { Cyclin D1 } \\
\text { and P27 } 27^{\text {KIP1 }}\end{array}$ & $\begin{array}{l}\text { A significant interaction was } \\
\text { detected between } p 27^{\mathrm{KIP1}} \text { and } \\
\text { treatment }(p=0.0049) \text {. Trastuzumab } \\
\text { effect was significant in the } p 27 \\
\text { KIP1 low subgroup }(p<0.001) \text {. No } \\
\text { trastuzumab effect was observed in } \\
\text { the } p 27^{\mathrm{KIP} 1} \text { high }(p=0.89) \text {. }\end{array}$ \\
\hline
\end{tabular}


glycogen synthase kinase-3 beta (GSK-3ß) ${ }^{14}$. On the other hand, GSK-3ß might phosphorylate cyclin D1 protein in the cytoplasm, preventing its association with protein required for nuclear import ${ }^{14}$. However, overexpression of canonical cyclin D1 protein alone is not sufficient to induce cancer transformation ${ }^{115,116}$.

It has been suggested that cyclin D1 is a multifaceted regulator that exists in two distinct isoforms, cyclin D1a and D1b. Cyclin D1a protein is rarely overexpressed in cancers, because it can be phosphorylated by GSK-3 $3 \beta^{117}$. However, cyclin D1b protein retains the ability to bind to and active CDK4, but the cyclin D1b protein is refractory to GSK-3ß nuclear export, and is thus, constitutively nuclear ${ }^{14}$. Expression of cyclin D1 mutant, cyclin D1b protein, which is not subject to GSK-3ß-dependent redistribution and remains in the nucleus during the cell cycle suggesting that deregulation of cyclin D1 nuclear export results in increased cyclin D1 oncogenic activity ${ }^{14,115}$. In addition, p2 $7^{\mathrm{KIP} 1}$ protein levels are reduced in cyclin D1b-expressing cells and that p2 $7^{\text {KIP1 }}$ binds cyclin D1b protein relatively poorly offers a possible explanation for why production of this isoform may be associated with increased cancer risk, based on the ability of cyclin D1b protein to evade restraint by $\mathrm{p} 27^{\mathrm{KIP} 1}$ as compared with cyclin D1a protein ${ }^{118}$. Therefore, expression of a non-phosphorylatable cyclin D1b protein may be sufficient to induce cell transformation ${ }^{115}$.

Furthermore, it has been suggested that cyclin D1a may merely be a marker of increased proliferation in cancer cells, but it may represent a key driver of oncogenesis in those who express cyclin D1b as well. Therefore, cyclin D1b may be the oncogenic mechanism that allows overexpression of cyclin D1a to function in an oncogenic capacity ${ }^{119}$. These studies and observations suggest that overexpression cyclin D1b and/or cyclin D1a can lead to strong nuclear cyclin D1 staining in OSCC cells. Finally, cyclin D1a retains the ability to bind to GSK-3ß and therefore it can be transported from nucleus to cytoplasm ${ }^{118}$. However, with a reduction in p2 $7^{\mathrm{KIP} 1}$ protein expression, cyclin D1a cannot be degraded in OSCC cells, therefore a strong cytoplasmic staining was observed in this study. Perhaps these could explain why we could observe strong nuclear and cytoplasmic cyclin D1 staining in OSCC specimen.

\section{CONCLUSION}

The mechanism of action of cyclins and cyclindependent kinases were discussed in this article. Cyclin D1 and $\mathrm{P} 27^{\mathrm{KIP} 1}$ have been identified as important regulators of cell cycle. The consensus amongst different research studies in literature is that cyclin D1 protein overexpression is associated with epithelial dysplasia and tumor progression. On the contrary, P27 ${ }^{\mathrm{KIP} 1}$ protein expression is decreased in cases with epithelial dysplasia and less differentiated grades of tumors. Therefore, both markers could be used as predictors for tumors' aggressiveness, prognosis, response to treatment and survival rates.

\section{CONFLICTS OF INTEREST}

None declared

\section{References}

1. Solomon MJ. Activation of the various cyclin/cdc2 protein kinases. Current Opinion in Cell Biology. 1993; 5(2): 180-6.

2. David OM. Principles of CDK regulation. Nature. 1995; 374(6518): 131-4.

3. Reed SI, Bailly E, Dulic V, et al. G1 control in mammalian cells. Journal of Cell Science. 1994; (Supp 18): 69-73.

4. Murray A. Cell cycle checkpoints. Current Opinion in Cell Biology. 1994; 6(6): 872-6.

5. Hunter T, Pines J. Cyclins and cancer II: Cyclin D and CDK inhibitors come of age. Cell. 1994; 79(4): 573-82.

6. Nigg EA. (1993). Targets of cyclin-dependent protein kinases. Current Opinion in Cell Biology. 1993; 5(2): 187-93.

7. Pines J. Cyclins and cyclin-dependent kinases: a biochemical view. Biochem J. 1995; 308: 697-711.

8. Morgan D. The cell cycle: principles of control. London: New Science Press. Sunderland, 2007.

9. Donnellan R, Chetty R. Cyclin D1 and human neoplasia. Molecular Pathology. 1998; 51(1): 1-7.

10. Motokura T, Bloom T, Kim HG, et al. (1991). A novel cyclin encoded by a bcl1-linked candidate oncogene. Nature. 1991; 350(6318): 512-5.

11. Han EK, Ng SC, Arber N, et al. Roles of cyclin D1 and related genes in growth inhibition, senescence and apoptosis. Apoptosis: an international journal on programmed cell death. 1999; 4(3): 213-219.

12. Coqueret O. Linking cyclins to transcriptional control. Gene. 2002; 299(1): 35-55

13. Diehl JA, Zindy F, Sherr CJ. Inhibition of cyclin D1 phosphorylation on threonine-286 prevents its rapid degradation via the ubiquitinproteasome pathway. Genes Dev. 1997; 11(8): 957-72.

14. Diehl JA, Cheng M, Roussel MF, et al. Glycogen synthase kinase-3beta regulates cyclin D1 proteolysis and subcellular localization. Genes Dev. 1998; 12(22): 3499-511.

15. Fracchiolla NS, Pruneri G, Pignataro L, et al. Molecular and immunohistochemical analysis of the bcl-1/cyclin D1 gene in laryngeal squamous cell carcinomas: correlation of protein expression with lymph node metastases and advanced clinical stage. Cancer. 1997; 79(6): 1114-21.

16. Buckley MF, Sweeney KJ, Hamilton JA, et al. Expression and amplification of cyclin genes in human breast cancer. Oncogene. 1993; 8(8): 2127-33.

17. Bartkova J, Lukas J, Müller H, et al. Cyclin D1 protein expression and function in human breast cancer. International Journal of Cancer. 1994; 57(3): 353-61.

18. Gillett C, Fantl V, Smith R, et al. Amplification and overexpression of cyclin D1 in breast cancer detected by immunohistochemical staining. Cancer research. 1994; 54(7): 1812-7

19. Bosch F, Jares P, Campo E, et al. PRAD-1/cyclin D1 gene overexpression in chronic lymphoproliferative disorders: a highly specific marker of mantle cell lymphoma. Blood. 1994; 84(8): 2726-32

20. Hayashi $T$, Ohno $H$, Yamabe $H$, et al. Clinical aspects of B-cell malignancy involving the BCL1/PRAD1 locus. International Journal of Hematology. 1994; 59(4): 281-96. 
21. de Boer CJ, Schuuring E, Dreef E, et al. Cyclin D1 protein analysis in the diagnosis of mantle cell lymphoma. Blood. 1995; 86(7): 2715-23.

22. Banks PM, Chan J, Cleary ML, et al. (1992). Mantle cell lymphoma. A proposal for unification of morphologic, immunologic, and molecular data. American Journal of Surgical Pathology. 1992; 16(7): 637-40.

23. Jiang W, Zhang YJ, Kahn SM, et al. (1993). Altered expression of the cyclin D1 and retinoblastoma genes in human esophageal cancer. Proceedings of the National Academy of Sciences of the United States of America. 1993; 90(19): 9026-30.

24. Naitoh H, Shibata J, Kawaguchi A, et al. Overexpression and localization of cyclin D1 mRNA and antigen in esophageal cancer American Journal of Pathology. 1995; 146(5): 1161-9.

25. Zhang YJ, Jiang W, Chen CJ, et al. Amplification and Overexpression of Cyclin D1 in Human Hepatocellular Carcinoma. Biochemical and Biophysical Research Communications. 1993; 196(2): 1010-6.

26. Nishida N, Fukuda Y, Komeda T, et al. Amplification and overexpression of the cyclin D1 gene in aggressive human hepatocellular carcinoma. Cancer Research. 1994; 54(12): 3107-10.

27. Ishikawa T, Furihata M, Ohtsuki Y, et al. Cyclin D1 overexpression related to retinoblastoma protein expression as a prognostic marker in human oesophageal squamous cell carcinoma. British Journal of Cancer. 1998; 77(1): 92-7.

28. Zhu XL, Hartwick W, Rohan T, et al. Cyclin D1 gene amplification and protein expression in benign breast disease and breast carcinoma. Modern Pathology. 1998; 11(11): 1082-8.

29. Mao Y, Li Z, Lou C, et al. Expression of phosphorylated Stat5 predicts expression of cyclin D1 and correlates with poor prognosis of colonic adenocarcinoma. International Journal of Colorectal Disease. 2011; 26(1): 29-35.

30. Michalides R, van Veelen N, Hart A, et al. Overexpression of cyclin D1 correlates with recurrence in a group of forty-seven operable squamous cell carcinomas of the head and neck. Cancer research. 1995; 55(5): 975-8.

31. Bartkova J, Lukas J, Müller H, et al. Abnormal patterns of D-type cyclin expression and G1 regulation in human head and neck cancer. Cancer research. 1995; 55(4): 949-56.

32. Kyomoto R, Kumazawa H, Toda Y, et al. Cyclin-D1-gene amplification is a more potent prognostic factor than its protein over-expression in human head-and-neck squamous-cell carcinoma. International Journal of Cancer. 1997; 74(6): 576-81.

33. Nakashima T, Kuratomi Y, Yasumatsu R, et al. The effect of cyclin D1 overexpression in human head and neck cancer cells. European Archives of Oto-Rhino-Laryngology. 2005; 262(5): 379-83.

34. Tashiro E, Maruki H, Minato Y, et al. Overexpression of cyclin D1 contributes to malignancy by up-regulation of fibroblast growth factor receptor 1 via the pRB/E2F pathway. Cancer research. 2003; 63(2): 424-31.

35. Meredith SD, Levine PA, Burns JA, et al. Chromosome 11q13 amplification in head and neck squamous cell carcinoma. Association with poor prognosis. Archives of otolaryngology-head \& neck surgery. 1995; 121(7): 790-4.

36. Gaffey MJ, Williams ME, Iezzoni JC, et al. Cyclin D1 (PRAD1, CCND1) and glutathione-S-transferase pi gene expression in head and neck squamous cell carcinoma. Human pathology. 1995; 26(11): 1221-6.

37. Akervall JA, Wennerberg JP, Michalides RJ, et al. Amplification of cyclin D1 in squamous cell carcinoma of the head and neck and the prognostic value of chromosomal abnormalities and cyclin D1 overexpression. Cancer. 1997; 79(2): 380-9.

38. Bova RJ, Quinn DI, Nankervis JS, et al. Cyclin D1 and p16INK4A expression predict reduced survival in carcinoma of the anterior tongue. Clinical Cancer Research. 1999; 5(10): 2810-19.
39. Mineta H, Miura K, Takebayashi S, et al. Cyclin D1 overexpression correlates with poor prognosis in patients with tongue squamous cell carcinoma. Oral Oncology. 2000; 36(2): 194-8.

40. Huang SF, Cheng SD, Chuang WY, et al. Cyclin D1 overexpression and poor clinical outcomes in Taiwanese oral cavity squamous cell carcinoma. World Journal Surgical Oncology. 2012; 10(1): 40.

41. Rousseau A, Lim MS, Lin Z, et al. Frequent cyclin D1 gene amplification and protein overexpression in oral epithelial dysplasias. Oral Oncology. 2001; 37(3): 268-75.

42. Castle JT, Cardinali M, Kratochvil FJ, et al. P53 and cyclin D1 staining patterns of malignant and premalignant oral lesions in agedependent populations. Oral surgery, oral medicine, oral pathology, oral radiology, and endodontics. 1999; 88(3): 326-32

43. Ramasubramanian A, Ramani P, Sherlin H, et al. Immunohistochemical evaluation of oral epithelial dysplasia using cyclin-D1, p27 and p63 expression as predictors of malignant transformation. Journal of Natural Science, Biology and Medicine. 2013; 4: 349-59.

44. Langenfeld J, Kiyokawa H, Sekula D, et al. Posttranslational Regulation of Cyclin D1 by Retinoic Acid: A Chemoprevention Mechanism. Proceedings of the National Academy of Sciences of the United States of America. 1997; 94(22): 12070-4.

45. Spinella MJ, Freemantle SJ, Sekula D, et al. Retinoic acid promotes ubiquitination and proteolysis of cyclin D1 during induced tumor cell differentiation. The Journal of biological chemistry. 1999; 274(31): 22013-8.

46. Yu Q Geng Y, Sicinski P. Specific protection against breast cancers by cyclin D1 ablation. Nature. 2001; 411(6841): 1017-21.

47. Alao JP, Lam EWF, Ali S, et al. Histone deacetylase inhibitor trichostatin A represses estrogen receptor alpha dependent transcription and promotes proteasomal degradation of cyclin D1 in human breast carcinoma cell lines. Clinical cancer research: an official journal of the American Association for Cancer Research. 2004; 10(23): 8094-104.

48. Huang JW, Shiau CW, Yang YT, et al. Peroxisome proliferatoractivated receptor gamma-independent ablation of cyclin D1 by thiazolidinediones and their derivatives in breast cancer cells. Molecular pharmacology. 2005; 67(4): 1342-8.

49. Alao J, Stavropoulou A, Lam E, et al. Histone deacetylase inhibitor, Trichostatin A induces ubiquitin-dependent cyclin D1 degradation in MCF-7 breast cancer cells. Molecular Cancer. 2006; 5(1): 8-19.

50. Morgan DO. Cyclin-dependent kinases: engines, clocks, and microprocessors. Annual Review of Cell Developmental Biology. 1997; 13: 261-91.

51. Sherr CJ, Roberts JM. Inhibitors of mammalian G1 cyclin-dependent kinases. Genes \& development. 1995; 9(10): 1149-63.

52. Parry D, Bates S, Mann DJ, et al. Lack of cyclin D-Cdk complexes in Rbnegative cells correlates with high levels of p16INK4/MTS1 tumour suppressor gene product. The EMBO journal. 1995; 14(3): 503-11.

53. Sandhu C, Garbe J, Bhattacharya N, et al. Transforming growth factor beta stabilizes p15INK4B protein, increases p15INK4Bcdk4 complexes, and inhibits cyclin D1-cdk4 association in human mammary epithelial cells. Molecular and Cellular Biology. 1997; 17(5): 2458-67.

54. Gartel AL, Radhakrishnan SK. Lost in transcription: p21 repression, mechanisms, and consequences. Cancer research. 2005; 65(10): 3980-5.

55. Chu IM, Hengst L, Slingerland JM. The Cdk inhibitor p27 in human cancer: prognostic potential and relevance to anticancer therapy. Nature Reviews Cancer. 2008; 8(4): 253-67.

56. Kato JY, Matsuoka M, Polyak K, et al. Cyclic AMP-induced G1 phase arrest mediated by an inhibitor (p27 Kip1) of cyclin-dependent kinase 4 activation. Cell. 1994; 79(3): 487-96. 
57. Polyak K, Lee MH, Erdjument-Bromage $\mathrm{H}$, et al. Cloning of $\mathrm{p} 27^{\mathrm{KIP}}$ a cyclin-dependent kinase inhibitor and a potential mediator of extracellular antimitogenic signals. Cell. 1994; 78(1): 59-66.

58. Slingerland JM, Hengst L, Pan CH, et al. A novel inhibitor of cyclinCdk activity detected in transforming growth factor beta-arrested epithelial cells. Molecular and Cellular Biology. 1994; 14(6): 3683-94.

59. Tsihlias J, Kapusta L, Slingerland J. The prognostic significance of altered cyclin-dependent kinase inhibitors in human cancer. Annual Review of Medicine. 1999; 50: 401-23.

60. Hengst L, Reed SI. Translational control of p27 $7^{\mathrm{KIP} 1}$ accummulation during the cell cycle. Science. 1996; 271(5257): 1861-4.

61. Sherr CJ, Roberts JM. CDK inhibitors: positive and negative regulators of G1-phase progression. Genes Dev. 1999; 13(12): 1501-12.

62. Liang J, Slingerland JM. Multiple roles of the PI3K/PKB (Akt) pathway in cell cycle progression. Cell cycle (Georgetown, Tex.). 2003; 2(4): $339-45$.

63. Besson A, Assoian RK, Roberts JM. Regulation of the cytoskeleton: an oncogenic function for cdk inhibitors? (cyclin-dependent kinase). Nature Reviews Cancer. 2004; 4(12): 948-55.

64. Payne SR, Zhang S, Tsuchiya K, et al. p27kip1 Deficiency Impairs G2/M Arrest in Response to DNA Damage, Leading to an Increase in Genetic Instability. Molecular and Cellular Biology. 2008; 28(1): 258-68.

65. Cordon-Cardo C, Koff A, Drobnjak M, et al. Distinct Altered Patterns of p27 Gene Expression in Benign Prostatic Hyperplasia and Prostatic Carcinoma. Journal of the National Cancer Institute. 1998; 90(17): 1284-91.

66. Tsihlias J, Kapusta LR, DeBoer G, et al. Loss of cyclin-dependent kinase inhibitor p27Kip1 is a novel prognostic factor in localized human prostate adenocarcinoma. Cancer Research. 1998; 58(3): 542-8.

67. Vis AN, Noordzij MA, Fitoz K, et al. Prognostic value of cell cycle proteins p27kip1 and mib-1, and the cell adhesion protein cd44s in surgically treated patients with prostate cancer. The Journal of Urology. 2000; 164(6); 2156-61.

68. Catzavelos C, Bhattacharya N, Ung YC, et al. Decreased levels of the cell-cycle inhibitor p27Kip1 protein: prognostic implications in primary breast cancer. Nature Medicine. 1997; 3(2): 227-30.

69. Moriya T, Sakamoto K, Sasano H, et al. Immunohistochemical analysis of Ki-67, p53, p21, and p27 in benign and malignant apocrine lesions of the breast: its correlation to histologic findings in 43 cases. Modern Pathology. 2000; 13(1): 13-8.

70. Oh YL, Choi JS, Song SY, et al. Expression of p21Waf1, p27Kip1 and cyclin D1 proteins in breast ductal carcinoma in situ: Relation with clinicopathologic characteristics and with p53 expression and estrogen receptor status. Pathology international. 2001; 51(2): 94-99.

71. de Paola F, Maria Vecci A, Maria Granato A, et al. (2002). p27/kip1 expression in normal epithelium, benign and neoplastic breast lesions. Journal of Pathology. 2002; 196(1): 26-31.

72. Thomas GV, Szigeti K, Murphy M, et al. Down-regulation of p27 is associated with development of colorectal adenocarcinoma metastases. Am J Pathol. 1998; 153(3): 681-7.

73. Zhang H, Sun XF. Loss of p27 expression predicts poor prognosis in patients with Dukes' B stage or proximal colorectal cancer International Journal of Oncology. 2001; 19(1): 49-52.

74. Bencivenga D, Caldarelli I, Stampone E, et al. p27Kip1 and human cancers: A reappraisal of a still enigmatic protein. Cancer Lett. 2017; 10(403): 354-365.

75. Yasui W, Kudo Y, Semba S, et al. Reduced expression of cyclindependent kinase inhibitor p27Kip1 is associated with advanced stage and invasiveness of gastric carcinomas. Japanese journal of cancer research: Gann. 1997; 88(7): 625-9.
76. Charles C, Nandita B, Yee CU, et al. Decreased levels of the cell-cycle inhibitor p27Kip1 protein: Prognostic implications in primary breast cancer. Nature Medicine. 1997; 3(2): 227-30.

77. Dreher T, Zentgraf H, Abel U, et al. Reduction of PTEN and p27kip1 expression correlates with tumor grade in prostate cancer. Analysis in radical prostatectomy specimens and needle biopsies. Virchows Arch. 2004; 444(6): 509-17.

78. Massimo L, Barry C, Sun WT, et al. Increased proteasome-dependent degradation of the cyclin-dependent kinase inhibitor p27 in aggressive colorectal carcinomas. Nature Medicine. 1997; 3(2); 231-4.

79. Mori M, Mimori K, Shiraishi T, et al. p27 expression and gastric carcinoma. Nature Medicine. 1997; 3(6): 593.

80. Aoyagi K, Kouhuji K, Miyagi M, et al. Expression of p27Kip1 protein in gastric carcinoma. Hepatogastroenterology. 2013; 60(122): 390-94.

81. Peggy LP, Kathleen EM, Patrick JH, et al. Expression of cell-cycle regulators p27Kip1 and cyclin E, alone and in combination, correlate with survival in young breast cancer patients. Nature Medicine. 1997; 3(2): 222-5.

82. Tan P, Cady B, Wanner M, et al. The cell cycle inhibitor p27 is an independent prognostic marker in small $(\mathrm{T} 1 \mathrm{a}, \mathrm{b})$ invasive breast carcinomas. Cancer Research. 1997; 57(7): 1259-63.

83. Shamma A, Doki Y, Tsujinaka T, et al. Loss of p27(KIP1) expression predicts poor prognosis in patients with esophageal squamous cell carcinoma. Oncology. 2000; 58(2): 152-8.

84. Ungermannova D, Parker SJ, Nasveschuk CG, et al. Identification and Mechanistic Studies of a Novel Ubiquitin E1 Inhibitor. Journal of Biomolecular Screening. 2012; 17: 421-34.

85. Xu J, Gimenez Conti IB, Cunningham JE, et al. Alterations of p53, cyclin $\mathrm{D} 1, \mathrm{Rb}$, and Hras in human oral carcinomas related to tobacco use. Cancer. 1998; 83: 204-12.

86. Lam KY, Ng IO, Yuen AP, et al. Cyclin D1 expression in oral squamous cell carcinomas: clinicopathological relevance and correlation with p53 expression. Journal of Oral Pathology and Medicine. 2000; 29(4): 167-72.

87. Nakahara Y, Shintani S, Mihara M, et al. Alterations of Rb, p16INK4A and cyclin D1 in the tumorigenesis of oral squamous cell carcinomas. Cancer Letters. 2000; 160(1): 3-8.

88. Ronaldo JB, David IQ, Janes S, et al. Cyclin D1 expression predicts reduced survival in early stage carcinoma of the anterior tongue. Australian Journal of OtoLaryngology. 2001; 4(1): 41-52.

89. Sathyan KM, Sailasree R, Jayasurya R, et al. Carcinoma of tongue and the buccal mucosa represent different biological subentities of the oral carcinoma. Journal of cancer research and clinical oncology. 2006; 132(9): 601-9.

90. Kövesi G, Szende B. Prognostic value of cyclin D1, p27, and p63 in oral leukoplakia. Journal of Oral Pathology \& Medicine. 2006; 35(5): 274-7.

91. Matsushima Y, Tsuji K, Tanaka A, et al. Immunohistochemical expression of cyclin D1 and cyclin B1 in oral leukoplakia. Journal of Cranio-Maxillofacial Surgery. 2006; 34(P210): 186.

92. Angadi PV, Krishnapillai R. Cyclin D1 expression in oral squamous cell carcinoma and verrucous carcinoma: correlation with histological differentiation. Oral Surgery, Oral Medicine, Oral Pathology, Oral Radiology and Endodontology. 2007; 103(3): e30-e35.

93. Uma S, Elizabeth J, Umadevi R, et al. Expression of p53 and Cyclin D1 in oral squamous cell carcinoma and normal mucosa: An Immunohistochemical study. Journal of Oral and Maxillofacial Pathology: JOMFP. 2012; 16(2): 172-7.

94. Liu Z, Long Y, Zhang Y, et al. Nuclear p27 expression confers a favorable outcome for nasopharyngeal carcinoma patients. Dis Markers. 2013;35(6):925-32. 
95. Li Y, Wei J, Xu C, et al. Prognostic significance of cyclin D1 expression in colorectal cancer: a meta-analysis of observational studies. PLoS One. 2014; 11;9(4): e94508.

96. Pereira RA, Ravinal RC, Costa RS, et al. Cyclin D1 expression in prostate carcinoma. Braz J Med Biol Res. 2014; 47(6):515-21.

97. Ravikumar G, Ananthamurthy A. Cyclin D1 expression in ductal carcinoma of the breast and its correlation with other prognostic parameters. J Can Res Ther 2014; 10:671-5.

98. Moreno-Galindo C, Hermsen M, García-Pedrero JM, et al. p27 and BCL2 expression predicts response to chemotherapy in head and neck squamous cell carcinomas. Oral Oncol. 2014; 50(2): 128-34.

99. Malgaonkar NI, Abuderman A, Kharma MY, et al. Evaluation of p27 Expression in Salivary Gland Neoplasms; A Step Forward in Unveiling the Role of p27. J Clin Diagn Res. 2016 Jul;10(7):ZC01-4.

100. Khabaz MN, Buhmeida A, Ghabrah T, et al. Cyclin D1 expression is associated with stage, grade and survival in urinary bladder carcinoma. Int J Clin Exp Med. 2016; 9(12): 23482-90.

101. Dhingra V, Verma J, Misra V, et al. Evaluation of Cyclin D1 expression in Head and Neck Squamous Cell Carcinoma. J Clin Diagn Res. 2017; 11(2): EC01-EC04.

102. Barić A, Marković V, Eterović D, et al. Cyclin D1, RET and P27 expression in papillary thyroid microcarcinoma. Acta Clin Croat. 2017; 56:15-20.

103. Patel SB, Manjunatha BS, Shah V, et al. Immunohistochemical evaluation of p63 and cyclin D1 in oral squamous cell carcinoma and leukoplakia. J Korean Assoc Oral Maxillofac Surg. 2017; 43: 324-330.

104. Guan S, Bakr MM, Firth N, et al. Expression of cyclin D1 correlates with p27KIP1and regulates the degree of oral dysplasia and squamous cell carcinoma differentiation. Oral Surgery, Oral Medicine, Oral Pathology, Oral Radiology and Endodontology. 2018; In Press.

105. Ramos-Garcia P, Bravo M, González-Ruiz L, et al. Significance of cytoplasmic cyclin D1 expression in oral oncogenesis. Oral Dis. 2018; 24(1-2): 98-102.

106. Filipits M, Dafni U, Gnant M, et al. Association of p27 and cyclin D1 expression and benefit from adjuvant trastuzumab treatment in HER2-positive early breast cancer: a TransHERA study. Clin Can Res. In Press. DOI: 10.1158/1078-0432.CCR-17-3473.

107. Rodolico V, Aragona F, Cabibi D, et al. Overexpression of cyclin D1 and interaction between p27Kip1 and tumour thickness predict lymph node metastases occurrence in lower lip squamous cell carcinoma. Oral Oncology. 2005; 41(3): 268-75.

108. de Andrade BA, Leon JE, Carlos R, et al. Immunohistochemical expression of p16, p21, p27 and cyclin D1 in oral nevi and melanoma. Head and Neck Pathology. 2012; 6(3): 297-304.

109. Perisanidis C, Perisanidis B, Wrba F, et al. Evaluation of immunohistochemical expression of p53, p21, p27, cyclin D1, and Ki67 in oral and oropharyngeal squamous cell carcinoma. Journal of Oral Pathology and Medicine. 2012; 41(1): 40-6.

110. Gillett C, Smith P, Gregory W, et al. Cyclin D1 and prognosis in human breast cancer. International Journal of Cancer. 1996; 69(2): 92.

111. de Falco M, Fedele V, De Luca L, et al. Evaluation of cyclin D1 expression and its subcellular distribution in mouse tissues. Journal of Anatomy. 2004; 205(5): 405-12.

112. Baldin V, Lukas J, Marcote MJ, et al. Cyclin D1 is a nuclear protein required for cell cycle progression in G1. 1993; Genes Dev. 7(5): 812-21.

113. Sewing A, Burger C, Brusselbach S, et al. Human cyclin D1 encodes a labile nuclear protein whose synthesis is directly induced by growth factors and suppressed by cyclic AMP. Journal of Cell Science. 1993; 104: 545-55.

114. Gladden AB, Diehl JA. Location, location, location: the role of cyclin D1 nuclear localization in cancer. Journal of Cell Biochemistry. 2005; 96(5): 906-13.

115. Alt JR, Cleveland JL, Hannink M, et al. Phosphorylationdependent regulation of cyclin D1 nuclear export and cyclin D1-dependent cellular transformation. Genes Dev. 2000; 14(24): 3102-14.

116. Quelle DE, Ashmun RA, Shurtleff SA, et al. Overexpression of mouse D-type cyclins accelerates G1 phase in rodent fibroblasts. Genes Dev. 1993; 7(8): 1559-71.

117. Burd CJ, Petre CE, Morey LM, et al. Cyclin D1b variant influences prostate cancer growth through aberrant androgen receptor regulation. Proceedings of the National Academy of Sciences USA. 2006; 103(7): 2190-95.

118. Lu F, Gladden AB, Diehl JA. An alternatively spliced cyclin D1 isoform, cyclin D1b, is a nuclear oncogene. Cancer Research. 2003; 63(21): 7056-61.

119. Abramson VG, Troxel AB, Feldman M, et al. Cyclin D1b in human breast carcinoma and co-expression with cyclin D1a is associated with poor outcome. Anticancer Research. 2010; 30(4): 1279-85. 